Ocular Oncology Update

\title{
Proton beam radiotherapy of uveal melanoma
}

\author{
Bertil Damato, MD, PhD, FRCOphth a,b,c,*; Andrzej Kacperek, BSc, $\mathrm{PhD}^{\mathrm{c}}$; Doug Errington, FRCR ${ }^{\mathrm{c}}$; \\ Heinrich Heimann, MD, FRCOphth ${ }^{\text {b }}$
}

\begin{abstract}
Proton beam radiotherapy of uveal melanoma can be administered as primary treatment, as salvage therapy for recurrent tumor, and as neoadjuvant therapy prior to surgical resection. The physical properties of proton beams make it possible to deliver highdoses of radiation to the tumor with relative sparing of adjacent tissues. This form of therapy is effective for a wider range of uveal melanoma than any other modality, providing exceptionally-high rates of local tumor control. This is particularly the case with diffuse iris melanomas, many of which are unresectable. The chances of survival, ocular conservation, visual preservation and avoidance of iatrogenic morbidity depend greatly on the tumor size, location and extent. When treating any side-effects and/or complications, it is helpful to consider whether these are the result of collateral damage or persistence of the irradiated tumor ('toxic tumor syndrome').
\end{abstract}

Keywords: Proton beam radiotherapy, Uveal melanoma, Recurrent tumor, Neoadjuvant therapy, Mortality

(C) 2013 Production and hosting by Elsevier B.V. on behalf of Saudi Ophthalmological Society, King Saud University. http://dx.doi.org/10.1016/j.sjopt.2013.06.014

\section{Introduction}

The aims of proton beam radiotherapy of uveal melanoma are to eradicate any threat of metastatic disease while conserving the eye with as much useful vision as possible.

As with other ionizing radiation, protons damage cells by disrupting DNA so that tumor cells lose their reproductive ability, hence entering senescence or undergoing apoptosis. ${ }^{1}$ Tissue damage is greatest at the point where the protons stop moving so that there is a 'Bragg Peak' of ionization, with relative sparing of healthy tissues both proximal and distal to the tumor target. Furthermore, the beam can be highly collimated, thereby reducing collateral damage to adjacent structures such as the optic nerve and fovea.
The aims of this review are to discuss the Liverpool approach to proton beam radiotherapy. ${ }^{2-4}$ This is done in the context of the current literature on the subject. ${ }^{5,6}$

\section{Methods}

\section{Initial assessment}

Initial investigations are aimed at determining the suitability of the patient for proton beam radiotherapy, estimating the likely outcomes, and planning treatment. The benefits and risks of all the therapeutic options are discussed. ${ }^{7}$ The tumor size and location are defined by slit-lamp examination

Available online 8 July 2013

a Ocular Oncology Service, Departments of Ophthalmology and Radiation Oncology, University of California, San Francisco, United States

b Ocular Oncology Service, Royal Liverpool University Hospital, United Kingdom

c Douglas Cyclotron Unit, Clatterbridge Cancer Centre, United Kingdom

* Corresponding author. Address: Ocular Oncology Service, Departments of Ophthalmology and Radiation Oncology, University of California, San Francisco, 10 Koret Way, K304, San Francisco, CA 94143-0730, USA. Tel.: +1 415514 1502; fax: +1 4154760336. e-mail address: DamatoB@Vision.UCSF.edu (B. Damato).
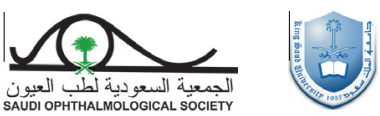

Peer review under responsibility of Saudi Ophthalmological Society, King Saud University 
and/or ophthalmoscopy as well as by color photography and ultrasonography. Secondary effects of the tumor on the eye, such as retinal detachment, are identified in addition to any unrelated, concurrent ocular disease. The axial length of the eye is measured to enable a computerized, 3D model of the eye to be generated. This is done using the EyePlan system developed at Clatterbridge.

\section{Insertion of tantalum markers}

If the tumor involves the choroid and/or ciliary body, then tantalum markers are inserted and these range from three to five in number, depending on the tumor shape and whether or not the optic disk can be used as the 'fourth marker' (Fig. 1). Unlike other authors, who perform a $360^{\circ}$ peritomy and who use muscle slings, we prefer to perform only a sectorial peritomy, involving only a third or half of the eye. With small, post-equatorial tumors, the overlying rectus muscle is temporarily dis-inserted to improve exposure and enhance accuracy. Before dividing any rectus muscles, the suture knot-to-limbus distances are measured and recorded; these measurements help to re-insert the muscle(s) more precisely by ensuring that the original knot-to-limbus distances are retained. The tumor margins are defined by transillumination and/or indentation. When performing transillumination, care is taken not to be misled by any penumbra, which can occur with transpupillary transillumination if the tumor is thick; in such cases, trans-ocular transillumination is superior. The first author (BD) has developed a right-angled 20-gauge transilluminator for this purpose. Marker-tumor, marker-marker and marker-limbus measurements are taken for each marker. For small, posterior tumors, Damato has devised a transilluminator that shines light through the eyes of the marker, which are visible with binocular indirect ophthalmoscopy (Altomed Ltd, Tyne and Wear, UK) (Fig. 2).

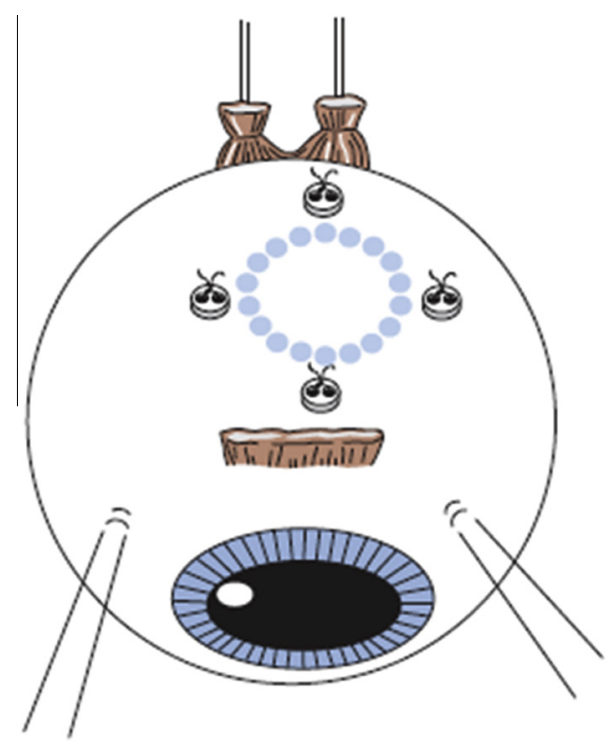

Insertion of tantalum markers

Figure 1. Drawing showing tantalum markers sutured to the sclera. The distances from the tumor margins, the limbus and from each other are measured. (From B. Damato, Ocular Tumours: Diagnosis and Treatment, Butterworth Heineman, 2000).
With iris melanomas and small ciliary body tumors with visible extension into the anterior chamber, the treatment can be administered safely without the use of tantalum markers.

\section{Simulation}

A 3D computer model of the eye is generated according to ocular biometry, tumor ultrasonography, color photography, intra-operative marker measurements as well as radiographs showing the marker positions (Fig. 3). As mentioned, with tumors involving iris, which are visible externally, markers are not required (Fig. 4). The model also shows the proton beam, the eyelids and the optic nerve. The direction of gaze is adjusted so as to minimize collateral damage to lens, optic nerve and fovea.

\section{Treatment}

A total dose of 53 to $70 \mathrm{~Gy}$ is administered in four, daily fractions. Some centers hyper-fractionate the treatment in the hope of reducing damage to healthy tissues. The patient's head is held in a frame, with a personalized face-mask and dental bite to ensure immobility. The position of the eye is maintained by means of a strategically-located visual target. Correct positioning of the eye is checked by radiography and by observing the eye with a closed-circuit TV during administration of the radiotherapy. The eyelids are retracted out of the field of the radiation beam. In Liverpool, if it is not possible for the upper eyelid to be pulled entirely out of the radiation field, the treatment is administered through the closed eyelid, with the patient gazing at the fixation target with the fellow eye.

With choroidal tumors, the proton beam radiotherapy is administered with safety margins of $2.0-2.5 \mathrm{~mm}$. To prevent collateral damage to the optic nerve and fovea, these margins can be reduced with small, juxtapapillary and juxtafoveal tumors if the patient is highly cooperative and if the measurements are known to be accurate. Ciliary body melanomas tend to develop diffuse spread circumferentially, which can be extensive if the tumor is large. Wider safety margins of up to $4 \mathrm{~mm}$ may therefore be used, in such cases.

With iris melanomas, we administer circumferential safety margins of $3 \mathrm{~mm}$ or one clock hour, whichever is greater, and with posterior safety margins extending $2 \mathrm{~mm}$ posterior to limbus if the tumor does not involve angle or $4 \mathrm{~mm}$ posterior to limbus if it does. If the iris tumor shows diffuse growth or seeding, then radiotherapy is delivered to the entire anterior segment with the beam extending posteriorly to ora serrata.

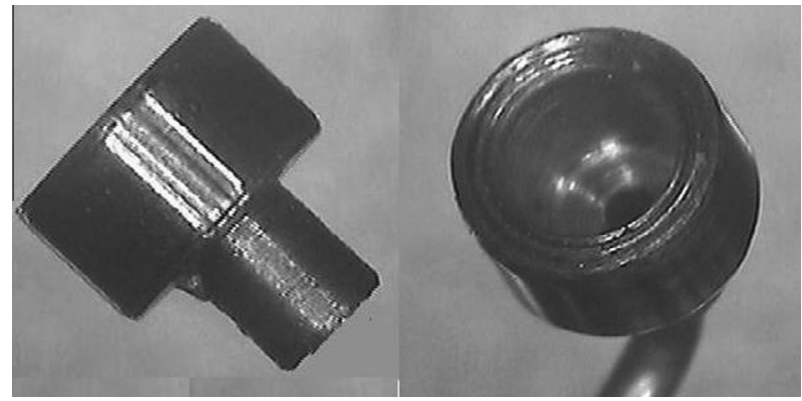

Figure 2. Transilluminator for localizing posterior tantalum markers, designed by the first author (BD). 


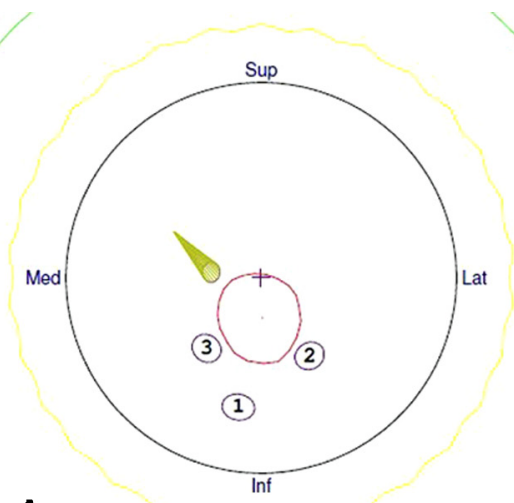

A

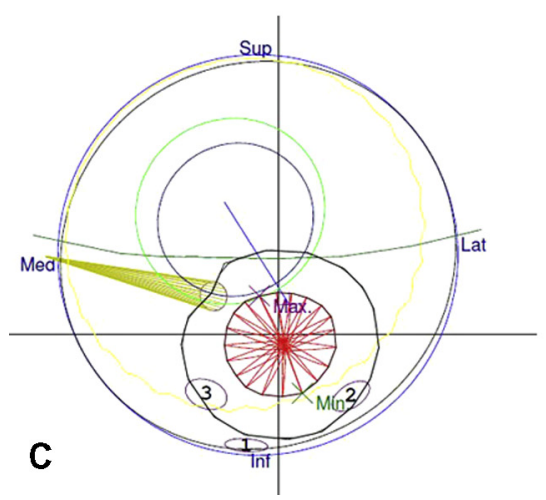

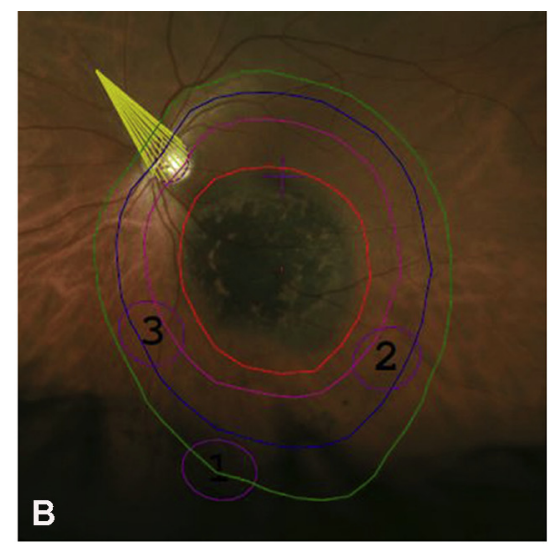

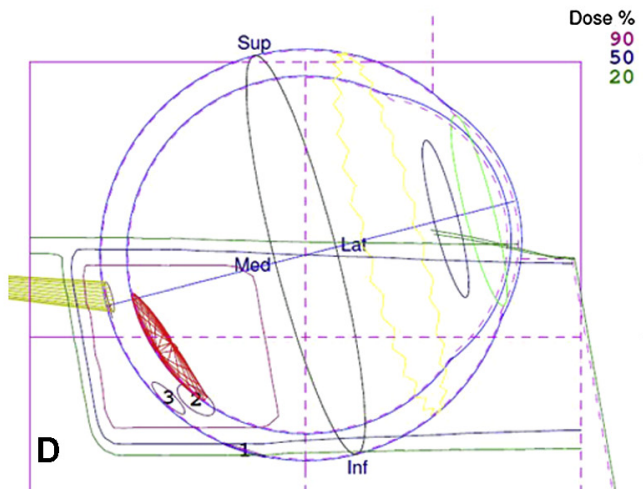

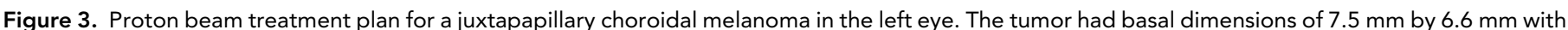

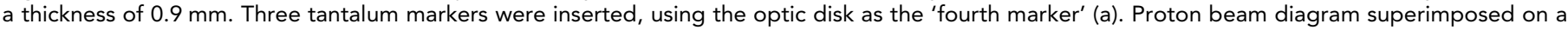

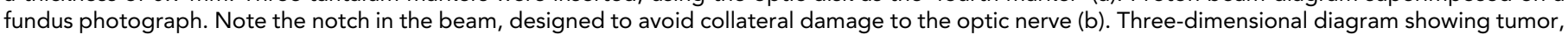
nerve, markers and beam (c). Vertical slice through eye showing minimal irradiation of the optic nerve (d).
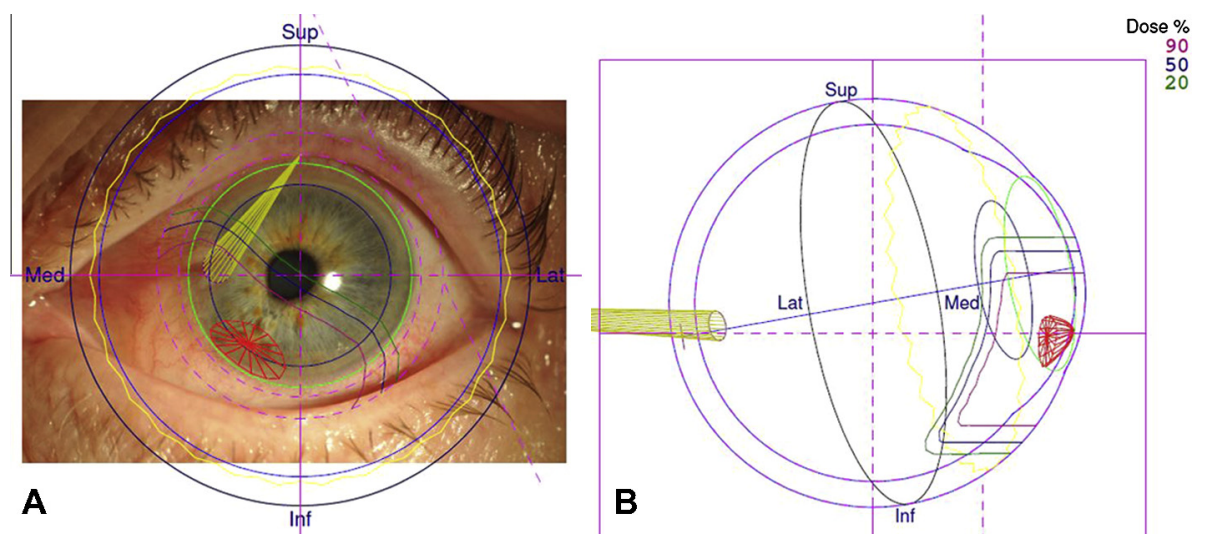

Figure 4. Infero-nasal iris melanoma in the left eye of a 74-year-old woman. The tumor had basal dimensions of $4.4 \mathrm{~mm}$ by $2.2 \mathrm{~mm}$ with a thickness of $1.6 \mathrm{~mm}$. Proton beam plan superimposed on the anterior-segment photograph (a). Horizontal section of the eye showing the penetration of the beam to the level of the ora (b).

\section{Biopsy}

Biopsy greatly enhances estimation of the survival probability by allowing the Tumor, Node and Metasis (TNM) Stage to be integrated with genetic and histologic data. Damato and associates have developed an online program for producing a personalized prognosis, also taking the patient's age and gender into account. ${ }^{8}$ This biopsy is performed after completion of the radiotherapy so as to avoid any risk of tumor seeding, and is done trans-retinally for choroidal tumors and trans-sclerally for ciliary body tumors.

\section{Long-term care}

After completion of the radiotherapy, the patient is monitored for any complications or side-effects. Long-term surveillance also includes screening for metastatic disease, either in selected patients having a poor prognosis or all patients. We prefer to screen selectively, according to survival estimates using an online program he has developed with associates in clinical engineering and pathology. This uses clinical, histologic and genetic data, also taking the patient's age and gender into account. Our preferred method is 
abdominal magnetic resonance imaging, performed every six or 12 months, according to the risk of metastasis.

\section{Counseling}

Counseling of patients and close relatives forms an essential part of care. At the initial visit, patients are informed of their condition, the natural history of uveal melanoma and the prognosis in terms of survival and ocular outcomes. The therapeutic options are discussed so that they can choose the optimal treatment for their particular condition and personality while considering their personal needs and fears. During the treatment, they are kept abreast of what is happening and what they might expect from day to day. After the proton beam treatment is completed, they are counseled repeatedly on their survival probability and on any ocular symptoms that might arise. Any stress or depression is managed, with the help of a health psychologist or specialist nurse. Feedback is obtained constantly so that any care can be personalized appropriately.

\section{Results}

Most centers administer proton beam radiotherapy similarly so that the outcomes are likely to be the same. Any differences in the results are likely to occur because of variations in patient selection and in the management of side-effects and complications. This article therefore focuses on our case series, which comprises 349 patients with choroidal melanoma, 88 patients receiving sectorial radiotherapy for iris melanoma and 12 patients undergoing proton beam radiotherapy of the entire anterior segment. ${ }^{2-4}$

\section{Local tumor control}

\section{Posterior segment tumors}

The cumulative risk of local treatment failure at 5 years is low (i.e. 1-6\%) (Fig. 5a). ${ }^{2}$ The strongest predictor of local recurrence is large tumor size. Larger tumors are more likely to show high-grade (i.e., aggressive) malignancy and to have sub-clinical, diffuse spread. In some cases, local recurrence can be treated by repeating the radiotherapy or by performing local resection or transpupillary thermotherapy. Survival after reirradiation is no worse than after secondary enucleation. ${ }^{10}$

\section{Iris melanomas}

The 5-year incidence of local tumor recurrence is approximately 6\% (95\% Confidence Interval, 0-14\%). ${ }^{3}$ Marginal tumor recurrence can develop after sectorial proton beam treatment if the extent of a diffuse melanoma is underestimated. Central tumor recurrences can occur but are rare, possibly arising if a narrow Bragg peak does not coincide with the tumor depth (unpublished cases). Such recurrent tumor can be treated by repeating the proton beam radiotherapy or by enucleation.

\section{Vision}

\section{Posterior segment melanomas}

The chances of conserving vision of 20/40, 20/200 and Count Fingers are approximately $32 \%, 42 \%$ and $73 \%$ at 8 years respectively (Fig. $5 \mathrm{~b}$ and c). ${ }^{2}$ The most important factors predicting visual loss are large tumor size, retinal invasion, ciliary body involvement, posterior tumor extension and reduced initial visual acuity. If the visual loss results from direct collateral damage to the disk or macula, then it is untreatable. ${ }^{11}$ Visual
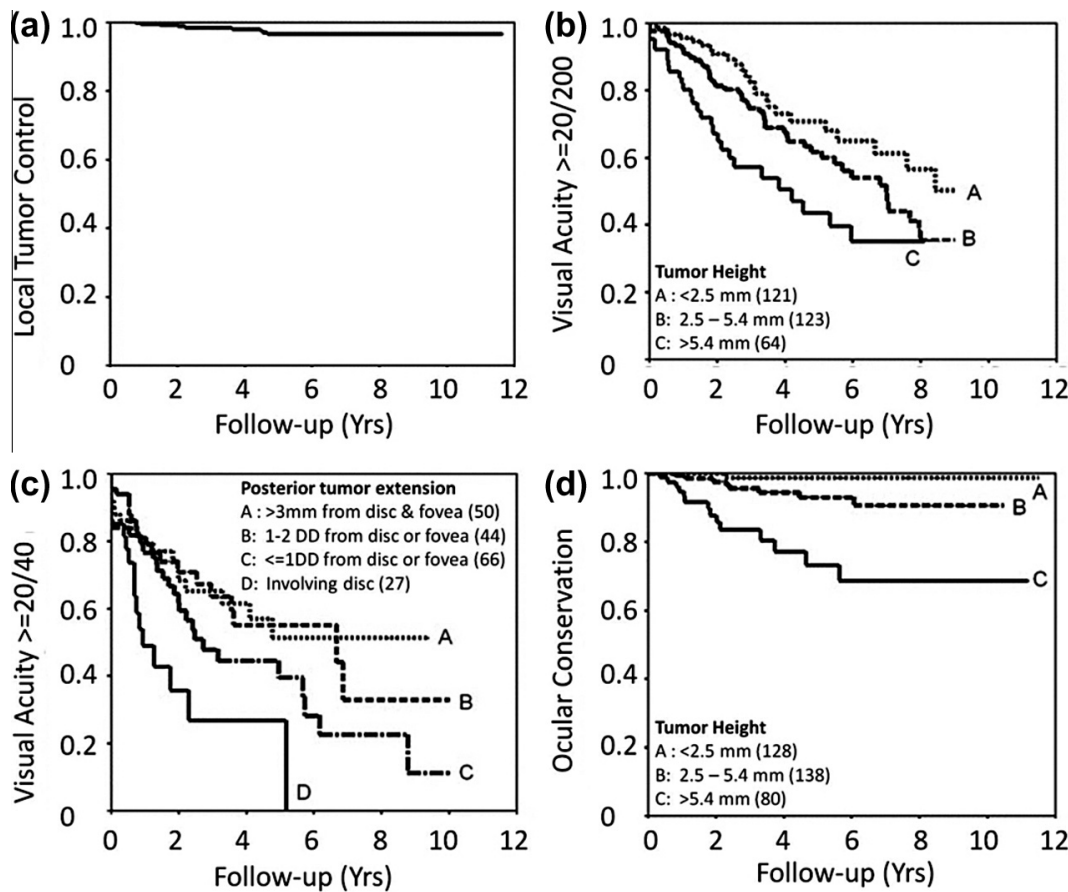

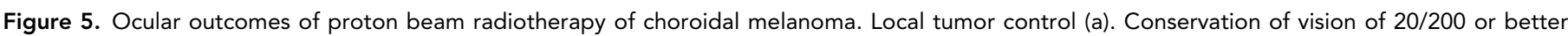

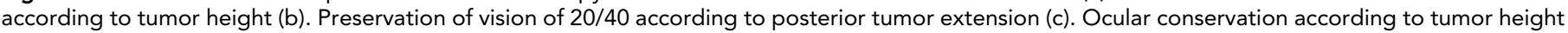

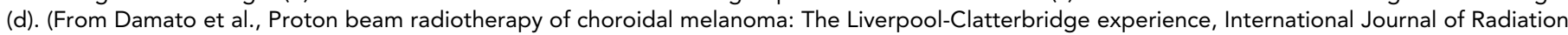
Oncology Biology Physics, Volume 62, Issue 5, 1 August 2005, Pages 1405-1411). 
loss caused by macular edema or retinal detachment may be managed by anti-angiogenic agents, intraocular steroid injections or implants and/or phototherapy or resection of the irradiated tumor. ${ }^{1}$ Any pre-treatment retinal detachment or edema involving the fovea can be treated by transpupillary thermotherapy or photodynamic therapy, delivered to the tumor immediately after completion of the radiotherapy. ${ }^{12}$

\section{Iris melanomas}

Most the eyes retain the pre-treatment visual acuity unless complications such as cataract or keratopathy develop (Fig. 6). ${ }^{3}$ Treatable causes of visual loss include cataract and hyphema. Cataract and keratopathy are treated in the usual manner. Recurrent hyphema may cease after photodynamic therapy of the irradiated tumor. ${ }^{13}$

\section{Ocular conservation}

\section{Posterior segment melanomas}

The risk of enucleation is approximately $5.4-14 \%$ at 5 years (Fig. $5 d$ ). ${ }^{2,14}$ The most common reasons for enucleation are neovascular glaucoma and local tumor recurrence. Risk factors include ciliary body involvement, posterior tumor extension close to disc, large tumor size and retinal invasion. Some authors advocate prophylactic anti-angiogenic agents as a means of preventing neovascular complications. The enucleation can be performed in the usual manner, using surgeon's preferred orbital implant.

\section{Iris melanomas}

The usual reason for secondary enucleation after proton beam radiotherapy of iris melanoma is local tumor recurrence. ${ }^{3}$ Another reason is painful keratopathy caused by limbal stem-cell deficiency if this does not respond to conservative measures. Neovascular glaucoma is rare.

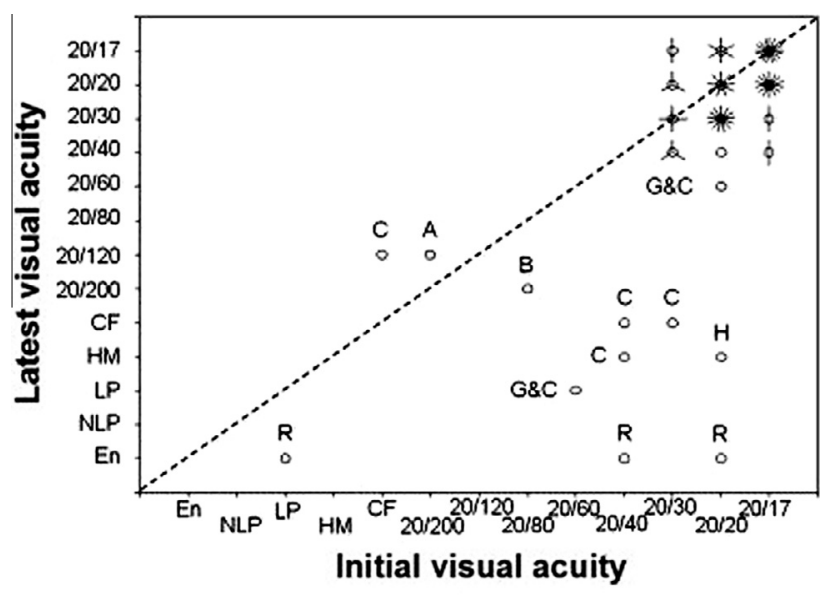

Figure 6. Visual acuity before proton beam radiotherapy of iris melanoma and at the latest known status. Each circle represents one case, and each line passing through this circle indicates an additional case. $\mathrm{A}=$ amblyopia; $\mathrm{B}=$ bullous keratopathy; $\mathrm{C}=$ cataract; $\mathrm{G}=$ glaucoma; $\mathrm{H}=$ hyphema; $\mathrm{R}=$ recurrence. (From Damato et al. Proton beam radiotherapy of iris melanoma, International Journal of Radiation Oncology Biology Physics, Volume 63, Issue 1, 1 September 2005, Pages 109-115).

\section{Miscellaneous ocular outcomes}

\section{Posterior-segment melanomas}

Irradiation of the eyelid causes an acute burn, which leaves a de-pigmented scar, with loss of lashes and squamous metaplasia of the tarsal conjunctiva with keratinization if the lid margin is affected. Such keratinization can cause pain and keratopathy if it occurs in the upper eyelid. It is managed by showing the patient how to debride the tarsus, removing the keratin with a cotton bud. Since 1993, we have prevented this complication by administering the proton beam radiotherapy through the closed eye if it is not possible to retract the eyelid entirely out of the radiation field. When the medial canthus is included in the beam, there may be canaliculitis, which results in intractable epiphora. Irradiation of the lacrimal gland can cause atrophy of this gland, with keratoconjunctivitis sicca. Other external eye complications include stem-cell deficiency in some patients receiving extensive limbal irradiation, an unstable tear film, and telangiectasia of the episcleral blood vessels. Subconjunctival markers can erode through irradiated conjunctiva to result in an indolent ulcer that is difficult to treat. This complication can be prevented by placing each marker outside the radiation field, or as far from the limbus as possible, or by removing the marker before it erodes through the conjunctiva.

Collateral damage to healthy intraocular tissues can cause cataract, retinal and choroidal vasculopathy involving the fovea, and optic neuropathy. Irradiated tumor tissue can become ischemic and/or exudative, resulting in macular edema, serous retinal detachment, retinal ischemia, rubeosis iridis and neovascular glaucoma. 'Toxic Tumor Syndrome'. The stimulus to neovascularization is enhanced by capillary closure in irradiated retina, optic neuropathy and detached retina, which becomes ischemic because of its separation from the choriocapillaris. The toxic tumor syndrome is managed by intraocular steroids or anti-angiogenic agents or by treatment of the irradiated tumor with phototherapy or resection. ${ }^{12,15}$ Rubeosis can also regress after intra-cameral or intravitreal injection of anti-angiogenic agents. ${ }^{16}$ Some authors have reported successful prevention of neovascular glaucoma by hyper-fractionation and/or delivering smaller doses of radiation to iris and ciliary body. ${ }^{17}$ Such results would indicate that neovascular complications can be the result of collateral damage to healthy tissues, at least in some patients.

If the tumor has perforated the retina, there can be recurrent vitreous hemorrhage. This can be treated by transpupillary thermotherapy or photodynamic therapy if there is a clear view after spontaneous resolution or vitrectomy. If these measures fail, then endoresection of the irradiated tumor may be considered.

Tumor biopsy can cause vitreous hemorrhage and, rarely, seeding of melanoma cells to other parts of the globe subconjunctivally. For these reasons, we prefer to perform this intervention after completion of the radiotherapy.

\section{Iris melanomas}

Cataract is common, especially if the tumor is extensive or if lens opacities are present before treatment. Glaucoma tends to occur as a result of annular tumor spread around the angle or by pigment scatter from a necrotic tumor. Such 
ocular hypertension can be managed with a drainage implant, since the tumor is sterile. ${ }^{18,19}$ Limbal stem-cell deficiency develops in approximately $30 \%$ of patients undergoing total anterior segment irradiation. Rarely, recurrent hyphema can arise from the telangiectatic tumor vessels, which can be treated by photodynamic therapy. ${ }^{13}$

\section{Survival}

\section{Posterior segment melanomas}

The risk factors for metastatic death are the same as for other forms of treatment and include large basal tumor diameter, thick tumor and extraocular tumor extension. ${ }^{2}$ If biopsy is performed, then epithelioid melanoma cytomorphology, chromosome 3 loss and class 2 gene expression profile would indicate an increased risk of metastasis. ${ }^{8}$ Local tumor recurrence is associated with increased mortality ${ }^{20}$; however, whether the recurrence causes the metastatic disease or whether it is merely an indicator of increased tumor malignancy is not known.

\section{Iris melanomas}

Data on survival after proton beam radiotherapy of iris melanomas are scanty. The general literature suggests that the most important factors predicting metastatic disease are diffuse tumor growth, secondary glaucoma (which correlates closely with diffuse growth), and large tumor size. ${ }^{21}$

\section{Discussion}

\section{Indications}

Of all therapeutic modalities, proton beam radiotherapy has the widest inclusion criteria. The placement of tantalum markers requires less surgical precision than plaque insertion, with both methods requiring accurate measurements for treatment planning. For these reasons, some centers rely exclusively on proton beam radiotherapy as a means of conserving the eye and vision. Only a few centers around the world have, however, have facilities for proton beam radiotherapy for ocular tumors.

The proton beam radiotherapy can be administered as primary treatment or as salvage therapy for local tumor recurrence after plaque radiotherapy, phototherapy or surgical resection. Some centers advocate neoadjuvant proton beam radiotherapy before endoresection as a means of preventing tumor seeding and metastasis. ${ }^{22}$ However, such radiotherapy frequently causes significant ocular morbidity and there is no evidence to show that it is indeed beneficial.

Even when plaque radiotherapy of uveal melanoma is possible, proton beam radiotherapy may be preferable when it reduces the chances of local tumor recurrence and/or collateral damage to the optic disk and/or fovea because the tumor has an awkward size, shape and/or location. With iris tumors, proton beam radiotherapy has more favorable dosimetry than brachytherapy and does not require any surgery.

\section{Contraindications}

If collateral damage to the optic disk is likely because the tumor extends within a disk diameter of the optic disk margin, then endoresection may be preferred if conservation of vision is highly desirable (e.g., in a monocular patient), if the patient accepts the controversial nature of such surgery, if the operation is unlikely to damage the fovea because of a medial tumor location, and if the surgeon is experienced in the technique. ${ }^{23}$ Concerns about tumor dissemination seem exaggerated. ${ }^{24}$

If the proton beam radiotherapy is likely to cause troublesome epiphora or if the toxic tumor syndrome is likely because the tumor is bulky or associated with an extensive retinal detachment, then trans-scleral tumor resection may be considered if the surgeon is experienced in the technique and if the patient is fit for hypotensive anesthesia. ${ }^{15}$

Retinopathy is more likely to develop in diabetic patients, who may need more intensive treatment for macular edema and other complications. As with other forms of conservative therapy, the patient must be sufficiently motivated to undergo what may be a prolonged course of treatment and followup; otherwise, enucleation may be more appropriate.

\section{Conclusions}

Proton beam radiotherapy of uveal melanoma achieves high rates of local tumor control and it is therefore likely that any influence on survival is similar to that of enucleation. Collateral damage to healthy ocular structures is predictable and eminently treatable in the case of cataract. The toxic tumor syndrome is less predictable but often treatable by a variety of methods. Any iatrogenic morbidity is more likely to be treated effectively if the pathology is better understood. As with any form of therapy, it is important to treat not only the tumor but the patient, addressing all needs and concerns by appropriate counseling and other psychological measures.

\section{Conflict of interest}

The authors declared that there is no conflict of interest.

\section{Acknowledgements}

The authors gratefully acknowledge Jenny Burke, Gary Cheetham, Gwendoline Hachuela, Gillian Hebbar, Julie Johnson, Samantha Kaye, Nicola Longrigg, Dawn Roberts, Martin Sheen, Julie Sudlow and Kathy Sztanko.

\section{References}

1. Groenewald C, Konstantinidis L, Damato B. Effects of radiotherapy on uveal melanomas and adjacent tissues. Eye (Lond) 2013;27(2):163-71.

2. Damato $B$, Kacperek A, Chopra M, Campbell IR, Errington RD. Proton beam radiotherapy of choroidal melanoma: the LiverpoolClatterbridge experience. Int $J$ Radiat Oncol Biol Phys 2005;62(5):1405-11.

3. Damato B, Kacperek A, Chopra M, Sheen MA, Campbell IR, Errington RD. Proton beam radiotherapy of iris melanoma. Int J Radiat Oncol Biol Phys 2005;63(1):109-15.

4. Konstantinidis L, Roberts D, Errington RD, Kacperek A, Damato B. Whole anterior segment proton beam radiotherapy for diffuse iris melanoma. Br J Ophthalmol 2013;97(4):471-4.

5. Gragoudas ES. Proton beam irradiation of uveal melanomas: the first 30 years. The Weisenfeld Lecture. Invest Ophthalmol Vis Sci 2006;47(11):4666-73.

6. Desjardins L, Lumbroso-Le Rouic L, Levy-Gabriel C, Cassoux N, Dendale R, Mazal A, et al. Treatment of uveal melanoma by accelerated proton beam. Dev Ophthalmol 2012;49:41-57.

7. Damato B, Heimann H. Personalized treatment of uveal melanoma. Eye (Lond) 2013;27(2):172-9. 
8. Damato B, Eleuteri A, Taktak AF, Coupland SE. Estimating prognosis for survival after treatment of choroidal melanoma. Prog Retin Eye Res 2011;30(5):285-95.

9. Marshall E, Romaniuk C, Ghaneh P, Wong H, McKay M, Chopra M, et al. MRI in the detection of hepatic metastases from high-risk uveal melanoma: a prospective study in 188 patients. $\mathrm{Br} \mathrm{J}$ Ophthalmol 2013;97(2):159-63.

10. Marucci L, Ancukiewicz M, Lane AM, Collier JM, Gragoudas ES, Munzenrider JE. Uveal melanoma recurrence after fractionated proton beam therapy: comparison of survival in patients treated with reirradiation or with enucleation. Int J Radiat Oncol Biol Phys 2011;79(3):842-6.

11. Lane AM, Kim IK, Gragoudas ES. Proton irradiation for peripapillary and parapapillary melanomas. Arch Ophthalmol 2011;129(9): 1127-30.

12. Damato $B$. Vasculopathy after treatment of choroidal melanoma. In: Joussen A, Gardner TW, Kirchhof B, Ryan SJ, editors. Retinal vascular disease. Berlin: Springer; 2007. p. 582-91.

13. Trichopoulos N, Damato B. Photodynamic therapy for recurrent hyphema after proton beam radiotherapy of iris melanoma. Graefes Arch Clin Exp Ophthalmol 2007;245(10):1573-5.

14. Egger E, Zografos L, Schalenbourg A, Beati D, Bohringer T, Chamot $L$, et al. Eye retention after proton beam radiotherapy for uveal melanoma. Int J Radiat Oncol Biol Phys 2003;55(4):867-80.

15. Damato BE, Groenewald C, Foulds WS. Surgical resection of choroidal melanoma. In: Ryan SJ, editor. Retina. 5th ed, Vol. 3. London: Elsevier; 2013. p. 2298-306.
16. Yeung SN, Paton KE, Waite C, Maberley DA. Intravitreal bevacizumab for iris neovascularization following proton beam irradiation for choroidal melanoma. Can J Ophthalmol 2010;45(3):269-73.

17. Daftari IK, Char DH, Verhey LJ, Castro JR, Petti PL, Meecham WJ, et al. Anterior segment sparing to reduce charged particle radiotherapy complications in uveal melanoma. Int $J$ Radiat Oncol Biol Phys 1997;39(5):997-1010.

18. Tan AN, Hoevenaars JG, Webers CA, Damato B, Beckers HJ. Baerveldt implant for secondary glaucoma due to iris melanoma. Clin Ophthalmol 2010;4:407-9.

19. Sharkawi E, Oleszczuk JD, Bergin C, Zografos L. Baerveldt shunts in the treatment of glaucoma secondary to anterior uveal melanoma and proton beam radiotherapy. Br J Ophthalmol 2012;96(8): 1104-7.

20. Egan KM, Ryan LM, Gragoudas ES. Survival implications of enucleation after definitive radiotherapy for choroidal melanoma: an example of regression on time-dependent covariates. Arch Ophthalmol 1998;116(3):366-70.

21. Shields CL, Kaliki S, Shah SU, Luo W, Furuta M, Shields JA. Iris melanoma: features and prognosis in 317 children and adults. J AAPOS 2012;16(1):10-6.

22. Bechrakis NE, Foerster $\mathrm{MH}$. Neoadjuvant proton beam radiotherapy combined with subsequent endoresection of choroidal melanomas. Int Ophthalmol Clin 2006;46(1):95-107.

23. Damato B, Groenewald C, McGalliard J, Wong D. Endoresection of choroidal melanoma. Br J Ophthalmol 1998;82(3):213-8.

24. Damato B. Choroidal melanoma endoresection, dandelions and allegory-based medicine. Br J Ophthalmol 2008;92(8):1013-4. 